\title{
Inkjet 3D Printing of Polymers Resistant to Fungal Attachment
}

\author{
Yinfeng He $\mathrm{H}^{1, \#, *}$, Cindy Vallières ${ }^{2, \#}$, Morgan R. Alexander ${ }^{3}$, Ricky D. Wildman ${ }^{1}$ and Simon V. Avery ${ }^{2, *}$
}

${ }^{1}$ Faculty of Engineering, University of Nottingham, Nottingham, UK; ${ }^{2}$ School of Life Sciences, University of Nottingham, Nottingham, UK; ${ }^{3}$ School of Pharmacy, University of Nottingham, Nottingham, UK

*For correspondence: Simon.Avery@nottingham.ac.uk; Yinfeng.He@nottingham.ac.uk

\#Contributed equally to this work

\begin{abstract}
[Abstract] Inkjet 3D printing is an additive manufacturing method that allows the user to produce a small batch of customized devices for comparative study versus commercial products. Here, we describe the use of a commercial 2D ink development system (Dimatix material printing) to manufacture small batches of 3D medical or other devices using a recently characterized fungal anti-attachment material. Such printed devices may resist problems that beset commercial medical products due to colonization by the fungal pathogen Candida albicans. By sequentially introducing the cross-section bitmaps of the product's CAD model and elevating the print head height using the auto-clicking script, we were able to create complex self-support geometries with the 2D ink development system. The use of this protocol allows researchers to produce a small batch of specimens for characterization from only a few grams of raw material. Additionally, we describe the testing of manufactured specimens for fungal anti-attachment. In comparison with most commercial AM systems, which require at least a few hundred grams of ink for printing trials, our protocol is well suited for smaller-scale production in material studies.
\end{abstract}

Keywords: Additive manufacturing, Inkjet, 3D printing, Candida albicans, Fungal biofilm

[Background] Additive manufacturing (AM) or 3D printing is a convenient tool for preparing small batches of products such as biomedical or electronic devices; however, commercial AM systems normally require at least a few hundred grams of material for the smallest batch of product, which remains a challenge for research focused on materials exploration. The Dimatix material printer is a commercial ink formulation development instrument designed for 2D printing, which runs with disposable cartridges that only require 3-5 grams of ink for printing. Its printing unit also allows height adjustment up to $25 \mathrm{~mm}$. With customized scripts, we were able to use this $2 \mathrm{D}$ printer to simulate an inkjet-based 3D printing process and prepare experimental specimens from only a few grams of raw material. This protocol has been used in our fungal anti-attachment material screening study (Vallieres et al., 2020), in which a batch of six specimens was produced using only 4 grams of ink made from the candidate monomer discovered in the materials screening. Biofilm formation by fungi such as Candida albicans is regarded as an important source of nosocomial systemic infections, with mortality rates up to $50 \%$ (Cavalheiro and Teixeira, 2018). The resistance of the printed materials to C. albicans colonization can be tested by measuring fungal biofilm formation using crystal violet, which binds to negatively charged surface molecules and polysaccharides in the extracellular matrix. The candidate anti-attachment methacrylate polymer, (R)- $\alpha$-acryloyloxy- $\beta, \beta$-dimethyl-y-butyrolactone) (AODMBA), 
was successfully printed into fungal-resistant voice prosthesis components, demonstrating the capability to use inkjet 3D printing to manufacture bespoke medical devices resistant to fungal attachment (Vallieres et al., 2020). In this paper, we detail the protocol for creating small batches of test specimens and subsequent testing against $C$. albicans. The preparation of inkjet-printable ink formulations and other key parameters for successful printing are described, as well as the scripts enabling the production of self-support 3D geometries. With the help of this protocol, material screening and specimen preparation can be simplified and accelerated for the discovery of printable materials for medical applications (e.g., in-dwelling devices resistant to fungal biofilms, as described here) or non-medical applications (e.g., identification of photoreactive materials for printing electronics).

\section{Materials and Reagents}

1. Starlab tips (Starlab, catalog numbers: S1111-1706 and S1111-6701)

2. $90 \mathrm{~mm}$ Petri dishes (Fisher Scientific, catalog number: 11308283)

3. $10 \mathrm{ml}$ and $25 \mathrm{ml}$ pipettes (Greiner Bio-One, catalog numbers: 607180 and 760180 )

4. $50 \mathrm{ml}$ centrifuge tubes (Scientific Laboratory Supplies, catalog number: SLS8110)

5. $2 \mathrm{ml}$ reaction tubes (Greiner Bio-One, catalog number: 623201)

6. 12-well and 96-well plates (Greiner Bio-One, catalog numbers: 665102 and 655185)

7. Nitrogen gas (BOC UN1066 compressed nitrogen)

8. $5 \mathrm{ml}$ syringe (BD Emerald REF 307731)

9. PEN film (GTS RPEN-075-0320)

10. Vials, $22 \mathrm{ml}$ disposable scintillation vials

11. Ductile tape, $\operatorname{Scotch}^{\circledR}$

12. Syringe filters, Millex-HA, MF-Millipore Membrane 50pk $0.45 \mu \mathrm{m}$

13. $0.2 \mu \mathrm{m}$ Sartorius ${ }^{\mathrm{TM}}$ Ministart ${ }^{\mathrm{TM}}$ syringe filter

14. Syringe, Braun inkjet $10 \mathrm{ml}$ syringe

15. BD Discardit II $20 \mathrm{ml}$ syringe

16. 2,2-Dimethoxy-2-phenylacetophenone (99\%, Sigma-Aldrich), stored at $4^{\circ} \mathrm{C}$

17. (R)- $\alpha$-acryloyloxy- $\beta, \beta$-dimethyl- $\gamma$-butyrolactone (AODMBA) (95\%, Sigma, catalog number: 376361), stored at $4^{\circ} \mathrm{C}$

18. RPMI 1640 with 20 mM HEPES and L-glutamine, without sodium bicarbonate (Sigma, catalog number: R7388)

NB: RPMI 1640 can be stored at $4^{\circ} \mathrm{C}$ for several months.

19. PBS (phosphate-buffered saline) (Fisher Scientific, catalog number: 10209252)

NB: PBS is autoclaved and stored at room temperature for several months.

20. Crystal violet (Sigma, catalog number: C3886)

NB: Crystal violet is made fresh each day and filter-sterilized using a BD Discardit II $20 \mathrm{ml}$ syringe and a $0.2 \mu \mathrm{m}$ Sartorius ${ }^{T M}$ Ministart $^{T M}$ syringe filter.

21. Isopropanol (Fisher Scientific, Laboratory reagent grade $\geq 99.5 \%$ ) 
22. YPD medium (see Recipes)

Bacteriological peptone (Oxoid, catalog number: LP0037)

Yeast extract (Oxoid, catalog number: LP0021)

D-glucose anhydrous (Fisher Scientific, catalog number: G/0500/61)

\section{Equipment}

1. $50 \mathrm{ml}$ Erlenmeyer flasks (Fisher Scientific, catalog number: 15499093)

2. PTFE stirrer $5 \mathrm{~mm}$

3. FujiFilm Dimatix DMP-2830 material printer

4. FujiFilm 10pL DMP cartridges DMC-1610/PN2100201146

5. Inert customized purge box PG-7-0444

6. UV unit: EPL UV 119-070 high-intensity unit

7. Stirring stage IKA RCT Basic

8. Fridge LEC Medical PE207C

9. Vacuum oven: Fistreem $31 \mathrm{~L}$ capacity

10. Gilson ${ }^{\mathrm{TM}}$ PIPETMAN ${ }^{\mathrm{TM}}$ Neo Pipets

11. Biological safety cabinet with UV unit

12. Heated incubator IN30 Memmert

13. Heated orbital shakers: New Brunswick ${ }^{T M}$ Innova $^{\circledR} 44$ (for flasks) ELMI SkyLine DTS-4 Digital Thermo Shaker (for microplates)

14. Eppendorf centrifuge 5810

15. Scientific Industries $\mathrm{SI}^{\mathrm{TM}}$ Vortex-Genie ${ }^{\mathrm{TM}} 2$

16. Hemocytometer Weber Scientific International Ltd.

17. Prior Scientific Microscope PX043

18. Sterile tweezers

19. BioTek EL800 Microplate Spectrophotometer

20. Balance (Denver Instrument SI-234)

\section{Software}

1. Fujifilm DMP version 2.0.0.1

2. GIMP 2.8.14

3. AutoHotKey 1.1 .32 .00

\section{Procedure}

Development of an ink for inkjet-based 3D printing processes should consider not only viscosity and surface tension as per typical 2D print ink formulations (Derby, 2010) but also the reactivity or 
solidification speed, which is important and correlates with factors such as photoinitiator concentration and printing environment. We have described these factors in detail in our previous studies ( $\mathrm{He}$ et al., 2016; Zhang et al., 2019). The protocol below revealed the successful candidate ink formulation developed from a (R)- $\alpha$-acryloyloxy- $\beta, \beta$-dimethyl- - -butyrolactone monomer printed in our recent work (Vallieres et al., 2020).

A. Ink preparation

1. Place a 5-mm PTFE stirrer into a 22-ml vial.

2. Place the vial on a balance and tare the weight.

3. Add $0.1 \mathrm{~g}$ 2,2-dimethoxy-2-phenylacetophenone (solid) into the vial.

4. Wrap the vial in aluminium film before adding liquid monomers.

5. Place the vial back on the balance and tare the weight.

6. Add $10 \mathrm{~g}(\mathrm{R})$ - $\alpha$-acryloyloxy- $\beta, \beta$-dimethyl- - -butyrolactone (liquid) to the vial and close the cap.

7. Place the vial on the stirring stage (IKA RCT Basic) and mix the contents at $800 \mathrm{rpm}$ for $15 \mathrm{~min}$ at room temperature.

8. Place the vial in a shaded box to help exclude ambient light during nitrogen purging. Open the cap and purge nitrogen gas (flow rate $\sim 10 \mathrm{ml} / \mathrm{min}$ ) into the mixture for $15 \mathrm{~min}$ through a needle, to allow the ink to become saturated with nitrogen. This step helps to minimize the oxygen inhibition effect during printing.

9. Collect the ink with a $10-\mathrm{ml}$ syringe in a dark room and purge through a syringe filter into a fresh vial. The filter will help to remove any solid contamination and avoid print head clogging during printing.

10. Cap the vial and wrap in aluminum foil to exclude ambient light that could trigger polymerization and cause ink solidification.

11. Store the ink for at least $12 \mathrm{~h}$ at $4^{\circ} \mathrm{C}$ before use.

B. Filling the ink cartridge

1. Carry out all the following steps in a dark room.

2. Carefully collect $3 \mathrm{ml}$ ink using a $5-\mathrm{ml}$ syringe, taking care to avoid the generation of bubbles at this stage.

3. Fix the flat-head needle that comes with the cartridge to the end of the syringe and carefully transfer the sampled $3 \mathrm{ml}$ ink into the cartridge bag.

4. Wrap the cartridge in ductile tape to avoid curing triggered by ambient light during printing and assemble the print head.

C. Printing and post-processing

1. Clamp the filled print head onto the DMP 2830 printer (Figure 1). 


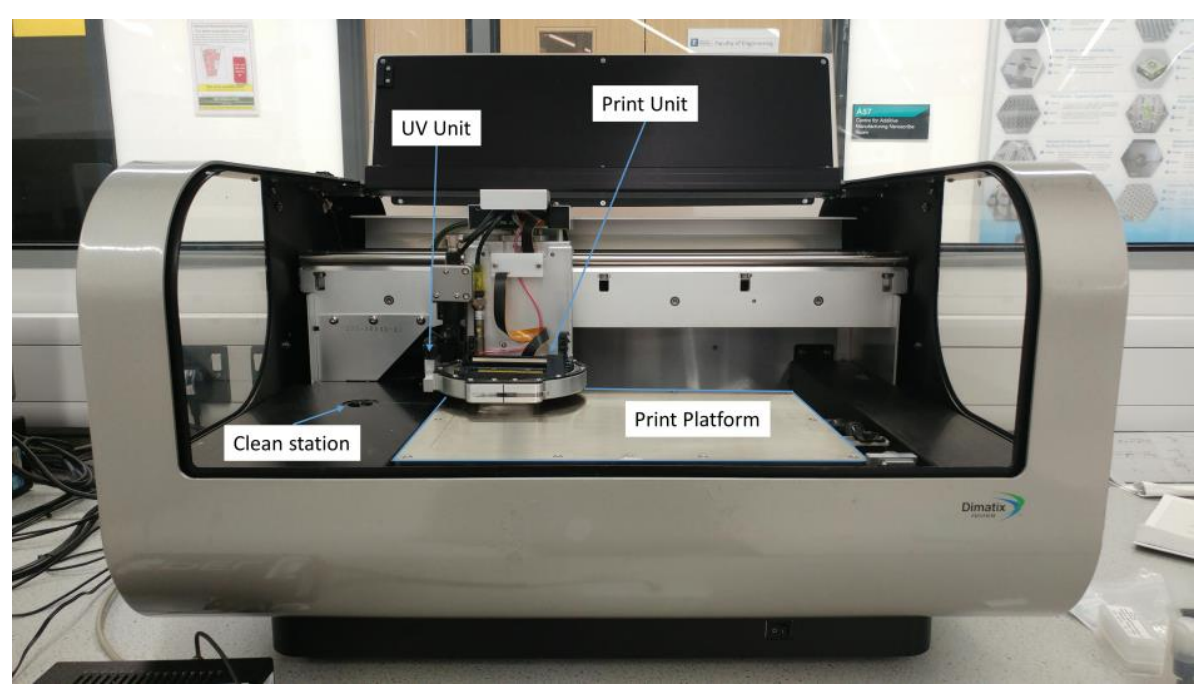

Figure 1. Interior set up of the Dimatix DMP 2830 printer

2. Affix the ceramic cleaning pad and purge the cartridge for $2 \mathrm{~s}$ to remove residual air from the print head.

3. Cut a PEN film to A4 size and clean both sides with isopropanol.

4. Place the film on the printing stage and turn on the vacuum to ensure that the film is firmly held on the stage.

5. Install the front panel of the glovebox and purge nitrogen until the oxygen level is reduced to $0.2-0.3 \%(\mathrm{v} / \mathrm{v})$.

6. Disable the tickle control function, monitor the drop viewer to check for droplet formation, and set the printing waveform as shown below (Table 1). 
Table 1. Key parameters of the jetting and non-jetting waveforms used in (R)- $\alpha-$ acryloyloxy- $\beta, \beta$-dimethyl-p-butyrolactone ink

\begin{tabular}{|c|c|c|c|c|c|}
\hline \multicolumn{6}{|c|}{ Jetting Waveform } \\
\hline & Level (\%) & Slew Rate & Duration $(\mu \mathrm{s})$ & $\begin{array}{l}\text { Maximum Jetting } \\
\text { Frequency (kHz) }\end{array}$ & $\begin{array}{l}\text { Waveform Width } \\
\text { ( } \mu \mathrm{s})\end{array}$ \\
\hline Section 1 & 0 & 0.65 & 3.584 & \multirow{4}{*}{5} & \multirow{4}{*}{11.52} \\
\hline Section 2 & 100 & 0.93 & 3.712 & & \\
\hline Section 3 & 13 & 0.6 & 3.392 & & \\
\hline Section 4 & 27 & 0.8 & 0.832 & & \\
\hline \multicolumn{6}{|c|}{ Non-Jetting Waveform } \\
\hline & Level (\%) & Slew Rate & Duration $(\mu \mathrm{s})$ & $\begin{array}{l}\text { Maximum Jetting } \\
\text { Frequency }(\mathrm{kHz})\end{array}$ & $\begin{array}{l}\text { Waveform Width } \\
(\mu \mathrm{s})\end{array}$ \\
\hline Section 1 & 33 & 1 & 3.712 & \multirow{3}{*}{5} & \multirow{3}{*}{11.52} \\
\hline Section 2 & 33 & 1 & 6.976 & & \\
\hline Section 3 & 27 & 1 & 0.832 & & \\
\hline
\end{tabular}

7. Set the printing temperature to $48^{\circ} \mathrm{C}$ and the meniscus to 4.0 inches $\mathrm{H}_{2} \mathrm{O}$. Adjust the printing voltage of each nozzle such that droplet formation is similar to that shown in Figure 2. The droplet speed needs to be between $7 \mathrm{~m} / \mathrm{s}$ and $10 \mathrm{~m} / \mathrm{s}$. Twelve jets (jets 5-16) were used in our work.

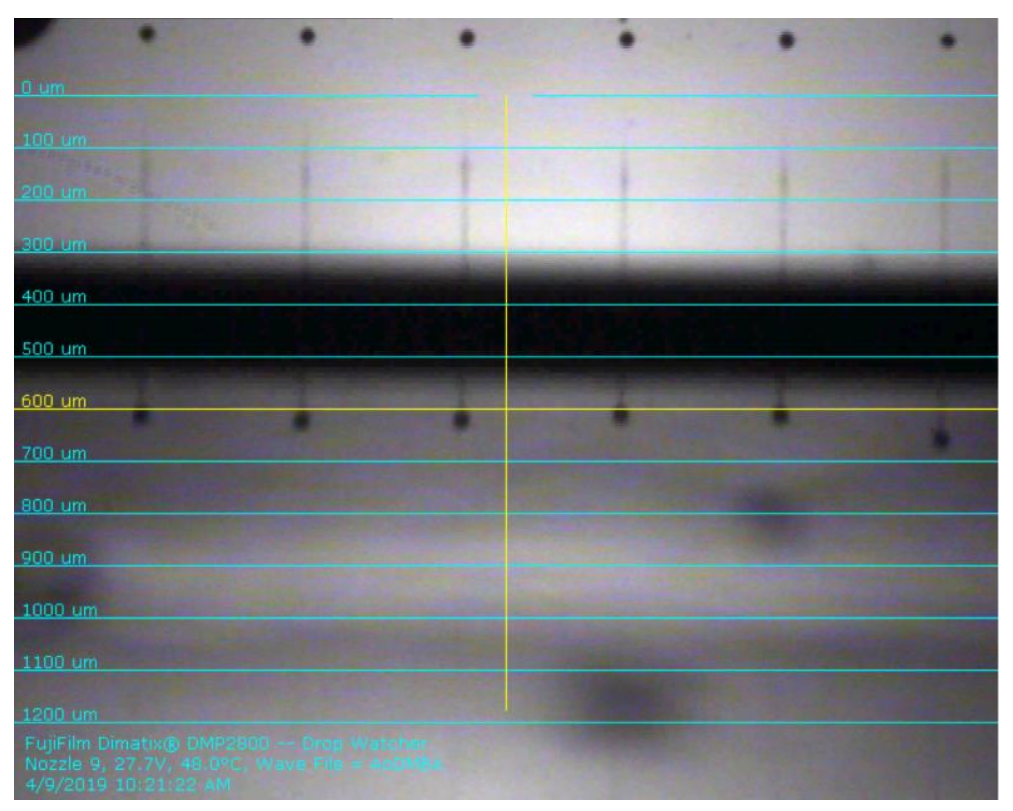

Figure 2. Exemplar droplet formation for (R)- $\alpha$-acryloyloxy- $\beta, \beta$-dimethyl- - -butyrolactone ink captured by the Dimatix instrument drop watcher. A full video is attached in the supplementary document.

8. Set the droplet spacing to $35 \mu \mathrm{m}$ and the print head angle to 5.8 degrees. 
9. Set the cleaning program to a $0.3 \mathrm{~s}$ purge and blot at the beginning of each layer, raising the print head by $12 \mu \mathrm{m}$ after each layer is finished.

10. The printing pattern for each layer is a mono bitmap, which is named as number+'.bmp' starting from 1.bmp; select 1.bmp in the printing program.

11. Copy the script in the supplement (Supplementary Script) into a notepad and save as an AHK file.

12. Run the saved script and sequentially input the 'original height', 'number of layers', and 'height increment per layer.' For (R)- $\alpha$-acryloyloxy- $\beta, \beta$-dimethyl- - -butyrolactone ink, we used $200 \mu \mathrm{m}$, 100 layers, and $11 \mu \mathrm{m}$ height increment per layer.

13. Deposit the liquid monomer ink onto the target location using a drop-on-demand inkjet print head. The printed ink is scanned and cured via the attached UV unit as it moves with the print head (He et al., 2017).

14. Collect the printed samples and place in a vacuum oven (room temperature, $-300 \mathrm{mmHg}$ ) for $24 \mathrm{~h}$ (Figure 3).

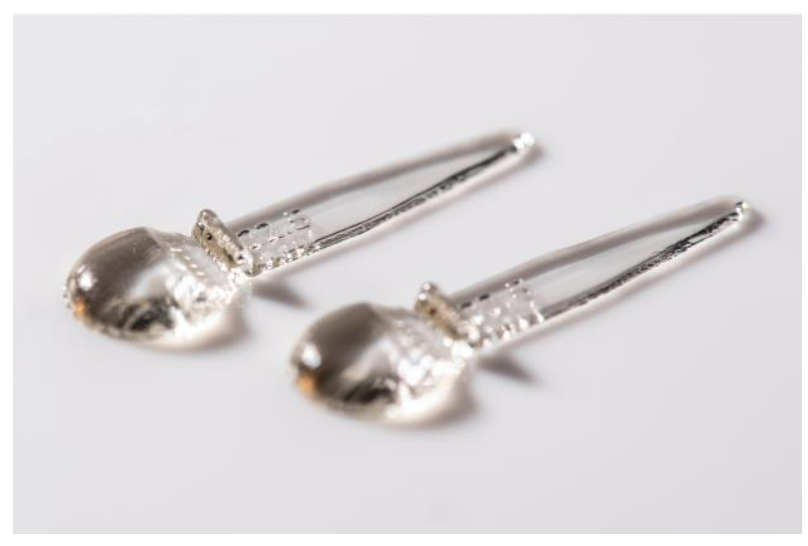

Figure 3. Voice prosthesis valve-flap samples collected after vacuum drying

15. Wash the samples by sequential immersion in isopropanol and distilled water, and dry in air.

16. Transfer the samples to a 12-well plate and irradiate with UV-C for 20 min to sterilize the samples.

D. Growing the fungal cultures, preparation of inocula, and biofilm formation (use aseptic technique; a biological safety cabinet may be required depending on the fungal species)

\section{Day 1 - Streak out strains}

1. Inoculate YPD agar medium with $C$. albicans cells from a stock kept at $-80^{\circ} \mathrm{C}$ (for the stock, freeze C. albicans cells at $-80^{\circ} \mathrm{C}$ in YPD broth with $20 \%$ glycerol) and incubate for 2 days in a static incubator at $37^{\circ} \mathrm{C}$. We used the $C$. albicans strain CAF2-yCherry; however, the method can be applied to other $C$. albicans strains.

\section{Day 3 - Set up overnight cultures}


2. Inoculate YPD broth medium (typically $10 \mathrm{ml}$ medium in a $50-\mathrm{ml}$ sterile Erlenmeyer flask) with a colony of $C$. albicans from a 2-day-old culture on YPD agar. We recommend assaying a minimum of three independent colonies. Incubate overnight in an orbital shaker (150 rpm) at $37^{\circ} \mathrm{C}$.

\section{Day 4 - Harvest cells and inoculate printed samples}

3. Harvest cells from the overnight liquid cultures by centrifugation $(3,000 \times g$ for $3 \mathrm{~min})$.

4. Remove the supernatant and wash once in $20 \mathrm{ml}$ PBS and once in $20 \mathrm{ml}$ RPMl 1640 using a 25-ml pipette.

5. Resuspend the final cell pellets in $10 \mathrm{ml}$ RPMI 1640 using a 10-ml pipette.

6. From the resulting cell suspensions, prepare 1:100 and/or 1:1,000 dilutions in RPMI 1640 in 2$\mathrm{ml}$ reaction tubes, vortex, and count using a hemocytometer under a brightfield microscope with $40 \times$ objective.

7. Calculate the inoculum required to prepare $40 \mathrm{ml}$ cell suspension at a final concentration of $1 \times$ $10^{6} \mathrm{ml}^{-1}$ cells in RPMI 1640.

8. Transfer $1 \mathrm{ml}$ adjusted cell suspension to each well of the 12-well plate (from Step C16).

9. Cover the plate with its lid, seal with tape, and incubate statically for $2 \mathrm{~h}$ at $37^{\circ} \mathrm{C}$ to allow the cells to adhere to the surface.

10. Use sterile tweezers to transfer the printed samples to a fresh plate and wash three times with $2 \mathrm{ml}$ PBS to remove non-adhered cells.

NB: Printed samples must be transferred carefully to fresh plates using tweezers to avoid mechanically disrupting cell attachment. The transfer of samples to fresh plates (here and in Step E2) is important since free cells can also adhere to the surface of the wells, which would add to the crystal violet signal intended to reflect biofilm formation on the printed sample, impairing the analysis.

11. Add $1 \mathrm{ml}$ fresh RPMI 1640 and incubate for $46 \mathrm{~h}$ at $37^{\circ} \mathrm{C}$ with orbital shaking at $100 \mathrm{rpm}$.

E. Fungal biofilm assessment (use aseptic technique and a biological safety cabinet)

Staining with crystal violet offers an approach for assessing the biomass of the biofilm. Alternatively, biofilm metabolic activity can be assayed; we detect such activity of $24 \mathrm{~h}$-biofilms by measuring the reduction of XTT (tetrazolium salt, 2,3-bis[2-methyloxy-4-nitro-5-sulfophenyl]-2H-tetrzolium-5carboxanilide). However, crystal violet is less expensive than XTT and allows for the easy visual detection of biofilms on the surface of samples (e.g., Figure 4C in Vallieres et al., 2020).

1. After $48 \mathrm{~h}, \mathrm{C}$. albicans should display mature biofilms. Aspirate the medium carefully so as not to touch or disrupt the biofilm.

2. Gently transfer the printed samples carrying biofilm to a fresh plate.

NB: As above, samples must be carefully transferred to fresh plates using tweezers to avoid mechanically disrupting the biofilm (mechanical disruption during sample transfer could lead to the biofilm detaching from the sample surface and interfering in the final assessment. Therefore, 
multiple specimens $(n>3)$ are required in each test to ensure that biofilm resistance is not a result of operational error. A control group (Vallieres et al., 2020) is also needed to ensure that the biofilm formation is as expected).

3. Stain the biofilms with $2 \mathrm{ml} 0.5 \%(\mathrm{w} / \mathrm{v})$ crystal violet for $1 \mathrm{~min}$ at room temperature.

4. Wash three times with $2 \mathrm{ml} \mathrm{PBS}$ to remove non-adhered biofilm and excess stain.

5. For quantitation, add $1 \mathrm{ml}$ ethanol to dissolve the crystal violet.

6. Transfer $100 \mu \mathrm{l}$ reaction to a 96-well plate. Dilutions of the reaction may be needed if the concentration of crystal violet is too high. We recommend the preparation of a standard curve to estimate the level of saturation of crystal violet absorption before the assay. To do so, measure the absorbance over a range of crystal violet (in ethanol) concentrations.

7. Measure the absorbance at $600 \mathrm{~nm}$ using the BioTek EL800 microtiter plate reader.

\section{Data analysis}

From the resulting colorimetric readings after subtracting the corresponding values for the negative control (ethanol only, i.e., no crystal violet), calculate the mean (of at least three biological replicates) and SEM, and plot the data. Alternatively, results for the 3D-printed samples can be presented as a percentage of the control [in Vallières et al., 2020, the positive control was a commercial prosthesis valve flap provided by Atos Medical (raw material Silastic Q7-4735, Dow Corning)].

\section{$\underline{\text { Notes }}$}

Crystal violet can be incompatible with some materials e.g., polyethylene glycol diacrylate $\left(\mathrm{PEG}_{575} \mathrm{DA}\right)$. Printed $\mathrm{PEG}_{575} \mathrm{DA}$ samples are completely stained with crystal violet even in the absence of $C$. albicans. For those materials, we recommend the assessment of fungal biofilm formation using the XTT reduction assay mentioned above.

\section{$\underline{\text { Recipes }}$}

1. YPD medium

$2 \%$ bacteriological peptone

$1 \%$ yeast extract

$2 \%$ D-glucose anhydrous

NB: Where necessary, the medium is solidified with $2 \%(w / v)$ agar (Sigma, catalog number: A7002). YPD medium is autoclaved and can subsequently be stored at room temperature for several months. 


\section{Acknowledgments}

This work was supported by the Biotechnology and Biological Sciences Research Council (grant number BB/P02369X/1) and the Engineering and Physical Sciences Research Council (grant numbers EP/N006615/1 and EP/N024818/1).

\section{Competing interests}

The authors declare no competing interests.

\section{$\underline{\text { References }}$}

1. Cavalheiro, M. and Teixeira, M. C. (2018). Candida Biofilms: Threats, Challenges, and Promising Strategies. Front Med (Lausanne) 5: 28.

2. Derby, B. (2010). Inkjet printing of functional and structural materials: fluid property requirements, feature stability, and resolution. Annu Rev Mater Res 40: 395-414.

3. He, Y., Wildman, R. D., Tuck, C. J., Christie, S. D., and Edmondson, S. (2016). An Investigation of the Behavior of Solvent based Polycaprolactone ink for Material Jetting. Sci Rep 6: 20852.

4. He, Y., Tuck, C. J., Prina, E., Kilsby, S., Christie, S. D., Edmondson, S, Hague R.J.M., Rose F.R.A.J., Wildman, R. D. (2017). A new photocrosslinkable polycaprolactone-based ink for three - dimensional inkjet printing. J Biomedical Mater Res B App Biomater 105(6): 1645-1657.

5. Vallieres, C., Hook, A. L., He, Y., Crucitti, V. C., Figueredo, G., Davies, C. R., Burroughs L., Winkler D.A., Wildman R.D., Irvine D.J., Alexander, M. R., Avery S.V., (2020). Discovery of (meth) acrylate polymers that resist colonization by fungi associated with pathogenesis and biodeterioration. Science Adv 6: eaba6574.

6. Zhang, F., Saleh, E., Vaithilingam, J., Li, Y., Tuck, C. J., Hague, R. J., Wildman R.D., He, Y. (2019). Reactive material jetting of polyimide insulators for complex circuit board design. Addit Manuf 25: 477-484. 